\title{
Simulating Single-Photon Sources Based on Backward-Wave Spontaneous Parametric Down-Conversion in a Periodically Poled KTP Waveguide
}

\author{
A.A. Shukhin, I. Z. Latypov ${ }^{\text {, }, ~ A . ~ V . ~ S h k a l i k o v, ~ a n d ~ A . ~ A . ~ K a l a c h e v ~}$ \\ Zavoisky Physical-Technical Institute, 10/7, Sibirsky tract str., 420029, Kazan, Russia
}

\begin{abstract}
The properties of the backward-wave spontaneous parametric down-conversion (SPDC) in a periodically poled potassium titanyl phosphate (KTP) waveguide are studied in the context of creating narrowband heralded sources of single-photon states. The effective index of refraction and spatial profile of different waveguide modes, efficiency of different SPDC processes and purity of heralded photons are calculated numerically for a given waveguide. Compared to the usual co-propagating SPDC, spectral narrowing of the backward-wave SPDC was observed as should be expected. Generation biphoton states in backward-wave regime is experimentally observed in two-photon detection scheme.
\end{abstract}

Keywords: single-photon source, SPDC, PPKTP

Developing narrowband sources of single photons and correlated photon pairs is an important part of quantum optics and quantum information [1]. In particular, single-photon wave packets of a sub-GHz or $\mathrm{GHz}$ bandwidth can effectively interact with atomic systems and therefore are demanded for implementation of quantum computing and quantum communication protocols involving optical quantum memories [2]. A promising approach to the problem is based on the backward-wave spontaneous parametric down-conversion (SPDC) in a nonlinear waveguide [3-5]. First, as with all SPDC-based sources, the photon number correlation between the down-conversion fields can be exploited to herald the existence of one photon by detection of its partner. In doing so, the vacuum state contribution is automatically removed from the output state while the multiphoton state contribution can be removed by using photon number resolving detectors. Second, emitting correlated photons in opposite directions allows one to reduce both SPDC bandwidth and spectral correlations within each photon-pair [3, 4]. As a result, it is possible to prepare single-photon states of high purity and $\mathrm{GHz}$ bandwidth at room temperature. Third, enclosing a nonlinear waveguide in a cavity allows one to realize SPDC in a single cavity mode of $\mathrm{MHz}$ bandwidth without additional filtering [5] thereby achieving high efficiency of the source. Finally, such a cavity-enhanced SPDC makes it possible to prepare single-photons of various wave forms by modulating the pump field [6].

We have studied the spectral properties of two-photon states and purity of heralded one-photon states produced by backward-wave spontaneous parametric down-conversion in a periodically poled KTP waveguide, which provides fifth order quasiphasematching for such a nonlinear interaction. Compared to the usual co-propagating SPDC regime in the same material, significant reduction of

${ }^{\text {a }}$ Corresponding author: ilnur.latypov@gmail.com 
SPDC bandwidth is observed. According to the numerical simulations, a single-photon source with the purity about 0.95 and bandwidth about $3 \mathrm{GHz}$ may be realized on the basis of the investigated material.

This research was supported by the Russian Science Foundation (Grant No. 14-12-00806).

\section{References}

1. M. D. Eisaman, J. Fan, A. Migdall and S. V. Polyakov, Rev. Sci. Instrum. 82 P.071101 (2011)

2. N. Sangouard, C. Simon, H. de Riedmatten and N. Gisin, Rev. Mod. Phys. 83 P. 33 (2011)

3. M. C. Booth, M. Atatüre, G. Di Giuseppe, B. E. A. Saleh, A. V. Sergienko and M. C. Teich, Phys. Rev. A 66 P. 023815 (2002)

4. A. Christ, A. Eckstein, P. J. Mosley and C. Silberhorn, Optics Express 17 P. 3441 (2009)

5. C. S. Chuu and S. E. Harris, Phys. Rev. A 83 P. 061803(R) (2011)

6. A. Kalachev, Phys. Rev. A 81 P. 043809 (2010) 